\title{
Identification of a bacteria-produced benzisoxazole with antibiotic activity against multi-drug resistant Acinetobacter baumannii
}

\author{
Robert W. Deering ${ }^{1} \cdot$ Kristen E. Whalen $\mathbb{1}^{2} \cdot$ Ivan Alvarez ${ }^{1} \cdot$ Kathryn Daffinee $^{3,4} \cdot$ Maya Beganovic $^{3,4}$.

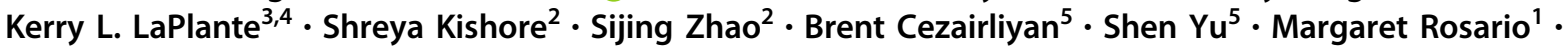 \\ Tracy J. Mincer ${ }^{6} \cdot$ David C. Rowley $\mathbb{B}^{1}$
}

Received: 29 October 2020 / Revised: 21 December 2020 / Accepted: 4 January 2021 / Published online: 12 February 2021

(c) The Author(s) 2021. This article is published with open access

\begin{abstract}
The emergence of multi-drug resistant pathogenic bacteria represents a serious and growing threat to national healthcare systems. Most pressing is an immediate need for the development of novel antibacterial agents to treat Gram-negative multidrug resistant infections, including the opportunistic, hospital-derived pathogen, Acinetobacter baumannii. Herein we report a naturally occurring 1,2-benzisoxazole with minimum inhibitory concentrations as low as $6.25 \mu \mathrm{g} \mathrm{ml}^{-1}$ against clinical strains of multi-drug resistant $A$. baumannii and investigate its possible mechanisms of action. This molecule represents a new chemotype for antibacterial agents against A. baumannii and is easily accessed in two steps via de novo synthesis. In vitro testing of structural analogs suggest that the natural compound may already be optimized for activity against this pathogen. Our results demonstrate that supplementation of 4-hydroxybenzoate in minimal media was able to reverse 1,2benzisoxazole's antibacterial effects in A. baumannii. A search of metabolic pathways involving 4-hydroxybenzoate coupled with molecular modeling studies implicates two enzymes, chorismate pyruvate-lyase and 4-hydroxybenzoate octaprenyltransferase, as promising leads for the target of 3,6-dihydroxy-1,2-benzisoxazole.
\end{abstract}

These authors contributed equally: Robert W. Deering, Kristen E. Whalen

Supplementary information The online version contains supplementary material available at https://doi.org/10.1038/s41429021-00412-7.

Kristen E. Whalen

kwhalen1@haverford.edu

$\bowtie$ Tracy J. Mincer

tmincer@fau.edu

$\triangle$ David C. Rowley drowley@uri.edu

1 Department of Biomedical and Pharmaceutical Sciences, College of Pharmacy, University of Rhode Island, Kingston, RI, USA

2 Department of Biology, Haverford College, Haverford, PA, USA

3 Department of Pharmacy Practice, College of Pharmacy, University of Rhode Island, Kingston, RI, USA

4 Infectious Diseases Research Program, Providence Veterans Affairs Medical Center, Providence, RI, USA

5 Octagon Therapeutics, Inc., Cambridge, MA, USA

6 Wilkes Honors College and Harbor Branch Oceanographic Institute, Florida Atlantic University, Boca Raton, FL, USA

\section{Introduction}

Acinetobacter baumannii is a non-fermenting, Gramnegative member of the Gammaproteobacteria commonly implicated in nosocomial sepsis, wound infections, and ventilator-associated pneumonia [1]. The World Health Organization recognizes antibiotic resistance as one of the three greatest threats to human health, and recently classified carbapenem-resistant $A$. baumannii as one of the most urgent threats, calling for a renewed investment in antibiotic development [2]. A. baumannii tenacity in hospitals is enhanced both by its ability to develop antibiotic resistance and capacity to survive on surfaces, including skin, for several days [3]. A. baumannii can also spread via aerosolization, promoting this pathogen's ability to easily colonize new environments [4-6]. A. baumannii is a member of the so-called 'ESKAPE' pathogens [7] and has garnered significant attention due to its propensity to cause wound infections in U.S. service members who served in Iraq and Afghanistan [8]. Alarmingly, intensive care unit patients contracting A. baumannii infections have a high mortality rate $(>50 \%)$, complicated by the fact that new pan-drug resistant (PR) strains of A. baumannii with 
resistance to all chemotherapeutic treatment options have now emerged [1, 8-10]. Carbapenems, typically in combination with other antimicrobial agents (e.g., the polypeptide colistin) are a commonly utilized treatment option for multidrug resistant (MDR) A. baumannii, but it is estimated that more than half of MDR strains are now carbapenem-resistant and colistin has significant toxicological and dosing concerns $[1,11,12]$. With the lack of new antibiotics in the drug discovery pipeline to treat Gramnegative infections, coupled with accelerated evolution of antibiotic resistance, it is imperative that new antibacterial drugs for treating A. baumannii infections be developed [13].

Although uncommonly reported from natural sources, benzisoxazole scaffolds are well represented in pharmacology, having shown significant importance in anti-HIV [14], antimicrobial [15-18], antipsychotic [19, 20], antiinflammatory [17, 21, 22], antioxidation [17, 23], anticancer [23-26], anticonvulsant [27] and antidiabetic [28, 29] research. Moreover, 1,2-benzisoxazole derivatives, which include zonisamide, risperidone, paliperidone, and iloperidone, are all FDA approved and currently in use for epilepsy, mood disorders, and schizophrenia, respectively [30-33].

Herein we report the identification of 3,6-dihydroxy-1,2benzisoxazole (1), a potent antibiotic against A. baumannii produced by a marine bacterium identified as a Bradyrhizobium denitrificans. A series of synthetically prepared analogs define key structural features for the antibacterial effects. We further provide evidence suggesting the mechanism of action (MOA) of 3,6-dihydroxy-1,2-benzisoxazole. In discovering 4-hydroxybenzoate (4-HB)'s ability to reverse the antibacterial property of $\mathbf{1}$, we investigate two possible 4-HButilizing target enzymes, chorismate pyruvate-lyase (CPL) and 4-HB octaprenyltransferase.

\section{Results}

\section{Bioassay-guided fractionation and dereplication of 3,6-dihydroxy-1,2-benzisoxazole}

Crude organic extract from the exudate of $B$. denitrificans (Isolate B158) was generated and initially screened in the $p$-iodonitrotetrazolium chloride (INT) assay to assess MDR reversal potential as described [34]. This initial screening demonstrated the extract from isolate B158 potentiated (i.e., reducing the antibiotic MIC by at least 4-fold) the activity of erythromycin when tested against $E$. coli MDR strains MG1655 $\triangle \mathrm{BC} / \mathrm{pABM}$, and MG1655 $\Delta \mathrm{BC} / \mathrm{pXYM}$. Phylogenetic analysis of $16 \mathrm{~S}$ rRNA gene sequence indicated that isolate B158 (Genbank Accession no. MF113387.1) was most closely related to B. denitrificans. Bioassay-guided fractionation of crude extract generated from 721 of B158 microbial culture resulted in the isolation of compound $\mathbf{1}$.

Compound $\mathbf{1}$ was isolated as an amorphous white powder. An $[\mathrm{M}-\mathrm{H}]^{-}$ion of 150.0204 using HRESIMS indicated a molecular formula of $\mathrm{C}_{7} \mathrm{H}_{5} \mathrm{NO}_{3} .{ }^{1} \mathrm{H}$ NMR resonances at $\delta_{\mathrm{H}} 7.49,6.75$, and 6.67 as well as the molecular formula suggested $\mathbf{1}$ to be 3,6-dihydroxy-benzisoxazole [35]. Because only $1 \mathrm{mg}$ of compound $\mathbf{1}$ was isolated from $14.1 \mathrm{~g}$ of crude extract, 3,6-dihydroxy-benzisoxazole was synthesized (Fig. S1) and determined to be identical to the natural product by NMR spectroscopy (Table S1) [35]. The synthetic derivative of compound $\mathbf{1}$ showed potent antimicrobial activity against Escherichia coli MDR strains AG102, MG1655 $\Delta \mathrm{BC} / \mathrm{pABM}$, and MG1655 $\Delta \mathrm{BC} / \mathrm{pXYM}$ with an MIC between 0.31 and $0.63 \mu \mathrm{g} \mathrm{ml}^{-1}$.

\section{3,6-Dihydroxy-1,2-benzisoxazole (1) inhibits the growth of clinically relevant strains of $A$. baumanii}

Compound 1 was previously reported to possess growth inhibitory effects against Gram-negative pathogenic bacteria, but not Gram-positives [36]. Because this prior testing was completed more than 30 years ago and did not include A. baumannii, we tested $\mathbf{1}$ for antibacterial activity against a panel of four clinically relevant A. baumannii strains (Table S2). Two strains (L1051 and NR-13382) were classified as carbapenem-resistant A. baumannii (CRAB) and as multi-drug resistant (MDR) organisms by having non-susceptibility to one or more agents in three or more antimicrobial categories using previously published guidelines [37, 38]. Minimum inhibitory concentrations (MICs) were determined to be in the range of $6.25 \mu \mathrm{g} \mathrm{ml}^{-1}$ to $50 \mu \mathrm{g}$ $\mathrm{ml}^{-1}$ with the most potent antibacterial effects against the MDR strains NR-13382 and L1051 (6.25 and $12.5 \mu \mathrm{g} \mathrm{ml}^{-1}$, respectively). While the MICs for these clinical strains were measured in Muller-Hinton broth (MHB), additional MIC testing was pursued using both MHB and DM01, a defined minimal medium supplemented with sodium pyruvate as a carbon source. We hypothesized that nutrient replete media could contain compounds that antagonized the antibiotic activity of $\mathbf{1}$, which could provide insight into compound $\mathbf{1}$ 's MOA. Interestingly, compound $\mathbf{1}$ was much more potent against multiple strains of Pseudomonas aeruginosa, E. coli, A. baumannii, and Klebsiella pneumoniae in assays using minimal media, DM01 (Table 1).

\section{Testing of synthetic analogs of 3,6-dihydroxy-1,2- benzisoxazole}

A panel of analogs was synthesized and purchased in order to probe the structural requirements necessary for the antibacterial effects of $\mathbf{1}$ as well as to potentially improve upon its potency (Fig. 1). None of the analogs (2-10) 
Table 1 MIC values for 3,6-dihydroxy-1,2-benzisoxazole (1) against Gram-negative bacteria in this study

\begin{tabular}{|c|c|c|}
\hline \multirow[t]{2}{*}{ Strain } & \multicolumn{2}{|c|}{$\mathrm{MIC}\left(\mu \mathrm{g} \mathrm{ml}^{-1}\right)$} \\
\hline & DM01 & MHB \\
\hline E. coli $\mathrm{ATCC} 25922^{\mathrm{TM}}$ & $0.25-0.5$ & $>500$ \\
\hline E. coli UNT 156 & 0.25 & 64 \\
\hline P. aeruginosa UCBPP14 & $8-16$ & $>500$ \\
\hline P. aeruginosa $\mathrm{PA} 27$ & 16 & 500 \\
\hline K. pneumoniae ATCC $43816^{\mathrm{TM}}$ & $>64$ & $>500$ \\
\hline K. pneumoniae ATCC BAA-2146 ${ }^{\mathrm{TM}}$ & $1-2$ & $>500$ \\
\hline A. baumannii UNT 190 & 2 & 128 \\
\hline A. baumannii UNT 197 & 2 & 16 \\
\hline A. baumannii L1051 & nt & 12.5 \\
\hline A. baumannii Isolate 9, NR-13382 & $\mathrm{nt}$ & 6.25 \\
\hline A. baumannii Naval-81, NR-17786 & nt & 25 \\
\hline A. baumannii АTCC $19606^{\mathrm{TM}}$ & $\mathrm{nt}$ & 50 \\
\hline
\end{tabular}

$n t$ not tested
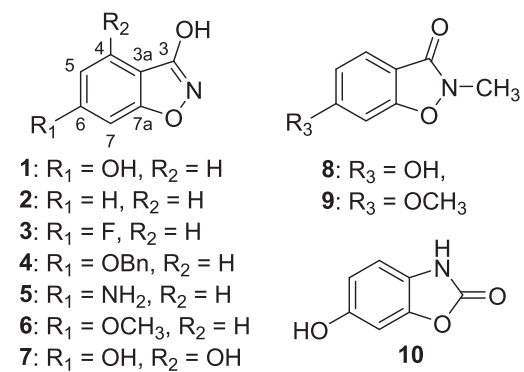

Fig. 1 Panel of compounds tested for MICs against A. baumannii in SAR study

demonstrated increased potency against the panel of clinical A. baumannii strains (Table S2). However, structural requirements for optimal potency were revealed. Replacement of the hydroxyl substituent at C6 (as in 2-6) displayed pronounced decreases in potency. Based on these data, a (H-bond) donor substituent at C6 appears to be required for antibacterial effects, with hydroxyl (1) as the preferred substituent over amino (5). Though the C6 methoxyl group (6) did not completely abolish activity, the dramatic decrease in potency concludes that alkyl modifications to the C6 hydroxyl group are unlikely to improve potency in this pharmacophore. Because of the preference of the hydroxyl substituent at the C6 position, an additional hydroxyl group was installed at C4 (7), but this abolished the antibacterial activity.

In addition to substituents on the benzene ring, the necessity of the isoxazole ring to the pharmacophore was investigated. Methylation of the nitrogen atom $(\mathbf{8}$ and $\mathbf{9})$ led to inactive products; it is noted that this modification leads to loss of conjugation in the hydroxyl-isoxazole ring.
Similarly, antibacterial activity was also completely absent from the oxazolone analog (10).

\section{4-Hydroxybenzoic acid antagonizes the antibacterial activity of 3,6-dihydroxy-1,2- benzisoxazole}

We next sought to more stringently test and better understand the mode of action of the active compound. The lower MICs observed in the minimal medium DM01 versus the replete growth medium MHB suggested that compounds in MHB might interfere with the antibiotic activity of $\mathbf{1}$. Growth characteristics of A. baumannii Ab197 and $P$. aeruginosa UCBPP14 (PA14) were measured using 96well phenotype microarray plates (PM1-PM5) purchased from Biolog, Inc. The bacteria strains were cultured in DM01 medium both with and without $2 \mu \mathrm{g} \mathrm{ml}^{-1}$ of compound 1 and growth was measured at 17 and $23 \mathrm{~h}$. These experiments revealed that 4-hydroxybenzoic acid (4-HB) and 4-hydroxybenzaldehyde antagonized the antibacterial effects of $\mathbf{1}$. To further examine this effect, PA14 was cultured in DM01, both with and without $16 \mu \mathrm{g} \mathrm{ml}^{-1}$ of $\mathbf{1}$ and 2-fold serial dilutions of 4-HB and 4-hydroxybenzaldehyde (Fig. S2). Both 4-hydroxybenzoic acid and 4hydroxybenzaldehyde antagonized the growth inhibitory effects of $\mathbf{1}$ at the three lowest concentrations tested $\left(62.5-250 \mu \mathrm{g} \mathrm{ml}^{-1}\right)$.

\section{Molecular docking of 3,6-dihydroxy-1,2- benzisoxazole onto $A$. baumannii CPL homology model}

The phenotypic array highlighted the antagonistic effect of 4-HB against 1, suggesting our benzisoxazole antibiotic may be targeting bacterial metabolic processes that involve 4-HB (Fig. 2). To examine if compound 1 has the potential to inhibit chorismate pyruvate-lyase (CPL), involved in the formation of 4-HB, we performed an in silico molecular docking study. In the absence of a crystal structure for A. baumannii CPL, E. coli (UniProt P26602) CPL was used to create a homology model. Superposition of the A. baumannii model with E. coli template shows a root-mean-square distance (RMSD) value of $0.780 \AA$ (Fig. S3), indicating a good fit to the model. Molecular docking under the solvated environment finds that compound 1 exhibits a favorable predicted binding affinity of $-5.8 \mathrm{kcal} \mathrm{mol}^{-1}$ (Table S3) and interacts with the primary site of A. baumannii model (Figs. 3 and S4). The binding of 4-hydroxybenzoate to $E$. coli CPL and compound $\mathbf{1}$ in A. baumannii involves five hydrogen bonds with four conserved residues (Arg76/Arg78, Glu155/Glu157, and peptide amide of Met34/Met35 and Leu114/Leu116) (Fig. 3). 


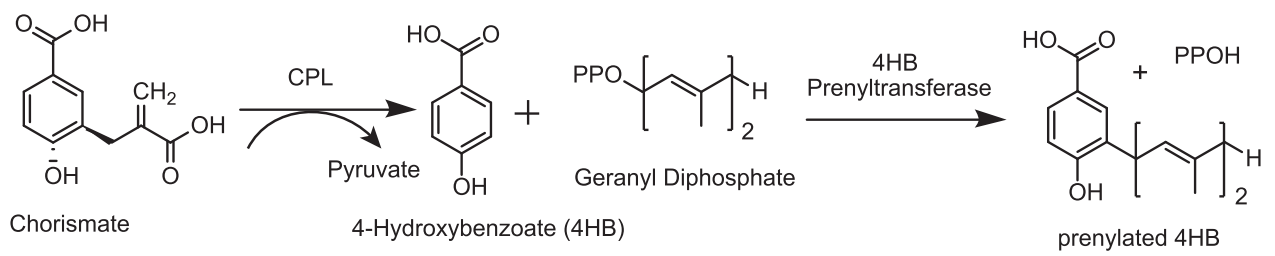

Fig. 2 Formation and utilization of 4-hydroxybenzoate by bacteria. Conversion of chorismate to 4-hydroxybenzoate (4HB) by chorismate pyruvate-lyase (CPL) in E. coli, followed by enzymatic synthesis of prenylated-4-hydrobenzoate catalyzed by $4 \mathrm{HB}$ octaprenyltransferase

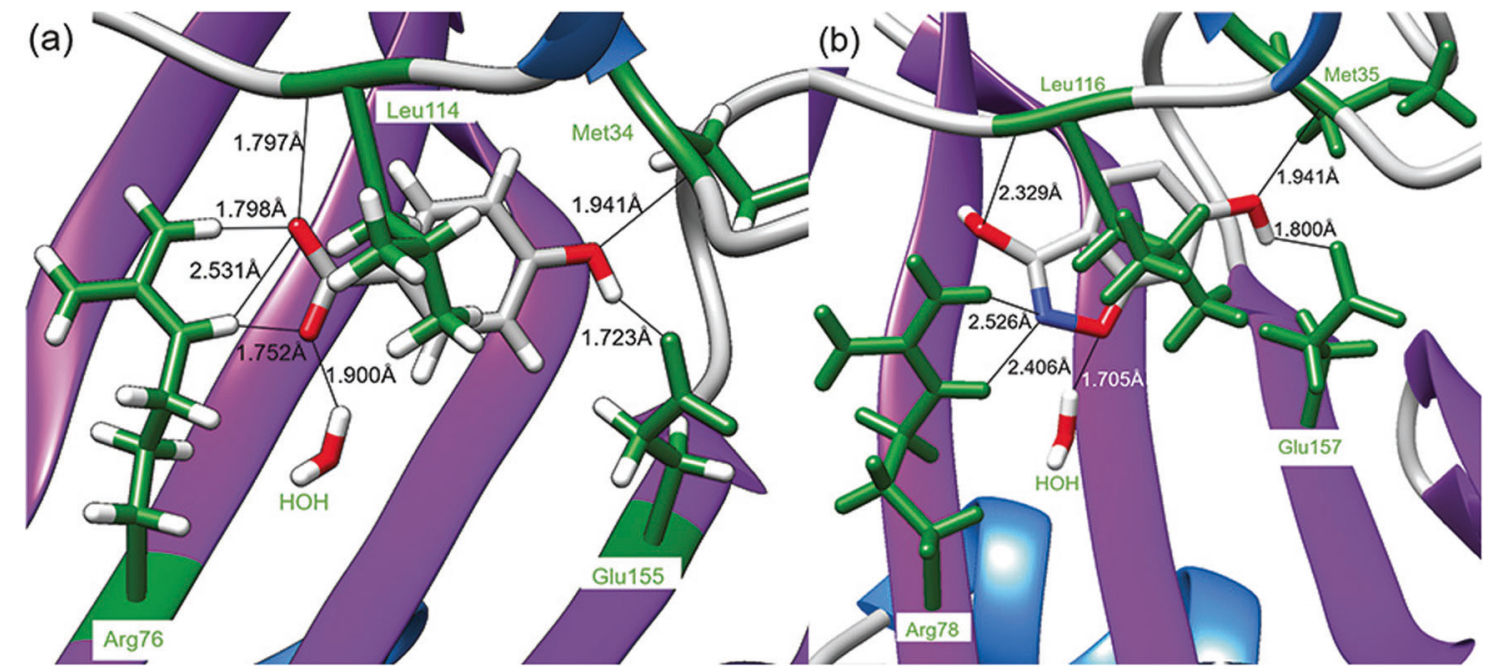

Fig. 3 Binding conformation of 4HB in E. coli CPL (PDB 1FW9) and compound 1 in A. baumannii (GenBank: SST05187.1) model. Hydrogen bonds (black lines) between ligand (gray) and protein residues (green) are labeled. a Binding of 4HB to E. coli CPL crystal structure (PDB:1FW9). b Molecular docking of compound $\mathbf{1}$ onto A. baumannii homology model under solvated (water) environment

\section{Discussion}

1,2-benzisoxazoles are bicyclic, heteroaromatic structures found in many approved drugs, are able to cross the blood-brain barrier, and have been classified as privileged structures for drug design for central nervous system disorders and other therapeutic areas [39, 40]. The heterocyclic ring structure of $\mathbf{1}$ shares similarity with the clinically used antibiotics cycloserine and linezolid. Interestingly, cycloserine was inactive against our panel of A. baumannii strains (data not shown). Oxazolidinone antibiotics are only effective against Gram-positive pathogens, and match the ring structure of the inactive 10, further supporting the highly specific nature of the 1,2-benzisoxazole pharmacophore for Gram-negative pathogens. Compound 1 was previously reported to possess potent growth inhibition of Gram-negative bacteria including E. coli, Proteus spp., Salmonella spp., K. pneumoniae, Enterobacter spp., and Serratia marcescens, as well as low toxicity with an $\mathrm{LD}_{50}$ of over $1500 \mathrm{mg}$ in mice $[35,36]$. Previous work indicated no inhibition was observed with the Gram-positive bacteria Staphylococcus aureus, Bacillus spp., Micrococcus sp., Corynebacterium sp., or the Gram-negative Pseudomonas spp. [35, 36].
From the panel of analogs tested, it appears that the structural features in the natural product $\mathbf{1}$ are necessary components of the antibiotic pharmacophore. A hydrogen bond donor is necessary at C6, and a hydroxyl is preferred over an amino group. Modifications to the heteroaromatic ring were found to be detrimental. Further optimization of the structure for antibacterial effects against $A$. baumannii would include determining if the 3-position is modifiable, as well as exploring other positions on the benzene ring for substitution.

Our results demonstrate that the antibacterial effect of $\mathbf{1}$ on $A$. baumannii can be reversed by supplementing the growth medium with 4-hydroxybenzoate (4-HB) and, to a lesser extent, by 4-hydroxybenzaldehyde. 4-HB is a precursor of ubiquinone biosynthesis for the electron transport chain and aerobic growth [41-43]. Connecting the antagonist effect of 4-HB with possible mechanisms of action, we have formulated two hypotheses pertaining to two target enzymes, CPL and 4-HB octaprenyltransferase, both of which are involved in the production and transformation of 4-HB (Fig. 2).

The production of 4-HB differs across animals and bacteria. Mammals synthesize 4-HB from tyrosine [41, 43], while Gram-negative bacteria produce 4-HB from chorismate, a product of the shikimate pathway [41, 44, 45]. 
In the first step of ubiquinone biosynthesis, bacterial CPL, the sole producer of 4-HB [46], irreversibly converts chorismate into 4-HB and pyruvate [46-48] (Fig. 2). The catalytic cycle of bacterial CPL involves a solvent accessible secondary binding site interconnected to an internal primary active site $[49,50]$, where product $4-\mathrm{HB}$ exhibits a ten-fold higher binding affinity than chorismate [47, 49-51]. This product inhibition mechanism allows 4-HB to limit ubiquinone production via a negative feedback pathway $[49,51,52]$ and stabilize CPL in the absence of chorismatebinding [50]. When chorismate binds at the secondary site, this entry encourages the release of 4-HB from the primary site, where chorismate subsequently enters and is converted into 4-HB and pyruvate [50]. Pyruvate leaves, while 4-HB remains bound to CPL for structural stability $[50,51]$. The CPL enzymes of four Gram-negative bacteria (E. coli, A. baumannii, K. pneumoniae, S. marcescens), which are all susceptible to 1 [35, 36], share between 83-100\% amino acid identity among the active site residues (Fig. S5), further bolstering a possible benzisoxazole-mediated binding to CPL. Furthermore, the lack of CPL in mammals may account for 1's low toxicity to mice [35, 36].

Our molecular docking results provide additional support of the CPL inhibition hypothesis, where compound $\mathbf{1}$ interacts with the primary site of CPL (Figs. 3 and S4). The five hydrogen-bonds and four conserved residues observed in the binding of 4-HB in E. coli are likewise maintained in compound 1's interaction with A. baumannii model, thereby indicating a similar binding conformation. Overall, the conserved binding interactions suggest that $\mathbf{1}$ may be a competitive product inhibitor of CPL, as a sufficient concentration of $\mathbf{1}$ may outcompete 4-HB at the primary binding site. The absence of 4-HB-binding may disrupt CPL's catalytic cycle $[50,51]$ and prevent the production of 4-HB, thus arresting ubiquinone pathway [46-48] and aerobic bacterial growth [41, 42].

Following CPL's production of 4-HB, membrane-bound 4-HB octaprenyltransferase (UbiA) prenylates 4-HB [41, 47, 48, 53], opening up yet a second speculation for compound 1's mechanism of action. 4-HB octaprenyltransferase is a membrane-bound enzyme in the ubiquinone biosynthesis pathway, encoded by the $u b i A$ gene in bacteria and by the COQ2 gene in mammals [41]. In the first step of the ubiquinone biosynthesis pathway, the bisubstrate enzyme binds both 4-HB and geranyl diphosphate to prenylate the meta position of 4-HB to yield 3-polyprenyl-4hydroxybenzoate (Fig. 2) [54]. A second hypothesis is then that 1 competitively inhibits 4-HB binding to UbiA, thereby preventing ubiquinone biosynthesis. Support for this second possibility comes from three main conditions: (i) 4-HB octaprenyltransferase is a promiscuous, non-specific enzyme towards both its substrates [41, 55] (ii) the necessity of a hydrogen bond donor at C6 for transfer of hydrogen to the negatively charged Asp191, which is theorized to result in the enhancement of the negative partial charge at the meta-position and hence activation of the benzene ring [54] (iii) the necessity of a functional group that is able to hydrogen bond at the position para to C6 in order to interact with Arg72 [54]. Both Asp191 and Arg72 are active site residues. Compound $\mathbf{1}$ satisfies both condition ii and iii and may mimic 4-HB, hindering prenylation either by blocking the active site or by getting prenylated itself. In the former case, ubiquinone cannot be formed without the prenyl tail. In the latter case, the prenylated products might not be able to proceed through the ubiquinone biosynthesis pathway due to the specificity of subsequent enzymes [56, 57]. A sequence alignment with $E$. coli's UbiA amino acid sequence (Fig. S6) demonstrated that Asp191 is conserved across three bacteria and two mammalian species, while Arg72 is conserved across E. coli, A. baumannii, $P$. aeruginosa and Mus musculus but not in humans. Hence, the active site $\operatorname{Arg} 72$ could enhance the specificity of $\mathbf{1}$ 's interaction in bacteria and lower mammals.

The ability to bind to CPL would also account for the reduced activity of the analogs $\mathbf{2 - 9}$. Compounds $\mathbf{2 - 4 , 6}$, and 9 all lack a C6 hydroxyl group and therefore would not enable H-bonding from C6 to the active site Asp191. Compounds 8 and 9 would lack the ability to form an Hbond from the $\mathrm{C} 3$ position to Arg72. With regard to compound 7 , it has been previously shown that substitutions to 4-HB at both ortho positions are not accepted as substrates for 4-HB octaprenyltransferase [55]. Compound 5, which substitutes an amine in place of the C6 hydroxyl group, retained modest activity, perhaps because it may still $\mathrm{H}$ bond with the active site residue Asp191. 4-HB octaprenyltransferase has previously been shown to tolerate a switch from hydroxy to an amino group in the 4-HB substrate [55].

It is interesting that the antibiotic effects of compound $\mathbf{1}$ are intensified under minimal media conditions. Marine bacteria frequently compete under minimal nutrient conditions for a good part of their life cycle [58]. Future studies to decipher the selective pressures that compound $\mathbf{1}$ are expressed under could yield more information as to the natural history of this molecule and allelopathic pathways in nature.

Narrow spectrum antibacterial drugs are becoming more desirable due to advances in diagnostic tests and changes to the regulatory environment [59]. Resistance is expected to develop more slowly to narrow-spectrum antibiotics and collateral damage to the gut microbiome, such as antibioticassociated colitis, is less likely [60]. Compound $\mathbf{1}$ was more active against $A$. baumannii strains than other Gramnegative bacteria when measured using standard MIC assays using Mueller-Hinton broth. Coupled with the current paucity of drugs available to treat MDR A. baumannii 
infections, we suggest that further studies to advance the development of 1,2-benzisoxazole antibiotics are warranted.

\section{Materials and methods}

\section{General experimental procedures}

NMR experiments were conducted using an Agilent NMRS $500 \mathrm{MHz}$ spectrometer with $\left(\mathrm{CD}_{3}\right)_{2} \mathrm{SO}$ (referenced to residual DMSO at $\delta_{\mathrm{H}} 2.50$ and $\delta_{\mathrm{C}} 39.5$ ) or $\mathrm{CD}_{3} \mathrm{OD}$ (referenced to residual $\mathrm{CH}_{3} \mathrm{OH}$ at $\delta_{\mathrm{H}} 3.31$ and $\delta_{\mathrm{C}} 49.0$ ) at $25^{\circ} \mathrm{C}$. High Resolution Electrospray ionization mass spectra (HRESIMS) were acquired using an $\mathrm{AB}$ Sciex TripleTOF 4600 spectrometer in the negative ion mode. Flash chromatography was completed with a Combiflash Rf200 equipped with a $40 \mathrm{~g}$ silica gel RediSepRf High Performance Gold column (Teledyne ISCO). HPLC experiments were performed on a Hitachi Elite LaChrom system equipped with a diode array detector (DAD) model L-2450, pump L-2130, and autosampler L-2200. Semi-preparative HPLC purifications were accomplished with a Waters XBridge Prep C18 $5 \mu \mathrm{m}, 10 \times 250 \mathrm{~mm}$ column at $4.5 \mathrm{ml}$ $\min ^{-1}$.

\section{Microbial isolation and identification}

A single comb jelly, Mnemiopsis leidyi, was obtained via collection by dip net at the surface from Eel Pond, Woods

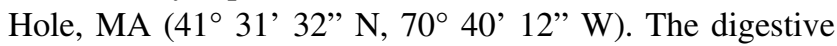
contents were stamped onto a tryptone seawater agar (TSW; $1 \mathrm{~g} \mathrm{l}^{-1}$ tryptone; 11 of 75:25 natural seawater:Milli-Q water; $15 \mathrm{~g}$ agar) plate and cultivated at $23{ }^{\circ} \mathrm{C}$ for three days. A single semi-transparent colony was transferred onto a fresh TSW plate to obtain a pure isolate of strain B158. The isolated strain B158 was identified to be B. denitrificans on the basis of $97.3 \%$ similarity in the 16S rRNA gene sequence $(2370$ nucleotides; Genbank Acc. No. MF113387.1) to B. denitrificans NBRC 105663 (Genbank Acc. No. AB682257.1). Pure stocks were frozen back in $10 \%$ DMSO and stored at $-80{ }^{\circ} \mathrm{C}$ until use.

\section{Bacteria culture and extract production}

A starter culture of B158 was prepared by inoculating $6 \mathrm{ml}$ of TSW media and with $100 \mu$ of frozen culture and incubated at $23{ }^{\circ} \mathrm{C}, 100 \mathrm{rpm}$ for 3 days. Large batch cultures to scale up growth consisted of 1.51 of TSY $\left(1 \mathrm{~g}^{-1}\right.$ tryptone, $1 \mathrm{gl}^{-1}$ yeast extract, 11 of 75:25 natural seawater: Milli-Q water) media inoculated with $1.5 \mathrm{ml}$ of 'starter' culture and grown at $100 \mathrm{rpm}$ for 8 days at $23^{\circ} \mathrm{C}$. Twentyfour hours before culture filtration (day 7), $20 \mathrm{ml}$ of 1:1 mixture of sterile, washed Amberlite XAD-7 and XAD-16 resin was added to the cultures. On the eighth day, the resin was filtered from the bacterial culture under vacuum, desalted by rinsing with Milli-Q water, and allowed to dry overnight at room temperature.

Metabolites were eluted from the resin first in $100 \mathrm{ml}$ of (1:1) methanol/dichloromethane, followed by $100 \mathrm{ml}$ of methanol. This extract was then dried under vacuum centrifugation (ThermoSavant). Dried extracts were stored at $-80{ }^{\circ} \mathrm{C}$ until bacterial susceptibility assays. In sum, 721 of B158 culture were processed as described above and yielded $14.5 \mathrm{~g}$ of crude extract. The crude extract was subjected to silica gel column chromatography with a step gradient of $100 \%$ isooctane, 4:1 isooctane/ethyl acetate; 3:2 isooctane/ethyl acetate, 2:3 isooctane/ethyl acetate, 1:4 isooctane/ethyl acetate, $100 \%$ ethyl acetate, 1:1 ethyl acetate/methanol, and 100\% methanol, yielding eight fractions. Active constituents, as determined with the INT assay, eluted in 100\% ethyl acetate and 1:1 ethyl acetate/ methanol (fractions 6 and 7) were combined (totaling $2374 \mathrm{mg}$ ) and further fractionated by SPE/ENVI-18 sep pak with a step gradient of 19:1 water/acetonitrile, 4:1 water/acetonitrile, 13:7 water/acetonitrile, 1:19 water/ acetonitrile, and $100 \%$ acetone. All solvents with the exception of acetone were acidified with $0.1 \%$ formic acid. Active constituents eluted in 4:1 water/acetonitrile and 13:7 water/acetonitrile. These fractions were combined (totaling $698.4 \mathrm{mg}$ ) and further chromatographed using a gradient of acetonitrile $(0.1 \%$ formic acid) and water ( $0.1 \%$ formic acid) by semipreparative HPLC carried out on an Agilent 1200 series equipped with an autosampler, diode array detector, quaternary pump, and 96-well plate fraction collector with a Phenomenex Luna $5 \mu \mathrm{m} \mathrm{C}_{18}(2)$, $100 \AA$, LC column $(250 \times 10 \mathrm{~mm})$ as the stationary phase with a flow rate of $4 \mathrm{ml} \mathrm{min}^{-1}$. Chromatography methods were as follows: begin at 5\% acetonitrile and ramp to $40 \%$ acetonitrile over $17 \mathrm{~min}$, ramp to $95 \%$ acetonitrile over $10 \mathrm{~s}$ and hold for $7 \mathrm{~min}$, return to $5 \%$ acetonitrile over $10 \mathrm{~s}$ and hold for $4 \mathrm{~min}$ at starting conditions. Active constituents eluted between 30-32\% acetonitrile with the resulting activity spread over two wells of a 96-well plate totaling $32.4 \mathrm{mg}$. The final purification of active fractions was achieved by using a gradient of methanol $(0.1 \%$ formic acid) and water $(0.1 \%$ formic acid) by analytical HPLC (Phenomenex Kinetex $2.6 \mu \mathrm{m}$ C18 $100 \AA$ A, LC column $(150 \times 2.1 \mathrm{~mm})$ as the stationary phase with a flow rate of $0.2 \mathrm{ml} \mathrm{min}^{-1}$ and UV detection at $276 \mathrm{~nm}$. Chromatography methods were as follows: being at 5\% methanol and hold for $5 \mathrm{~min}$, ramp to $40 \%$ methanol over $11 \mathrm{~min}$, hold at $40 \%$ methanol for $1 \mathrm{~min}$, ramp up to $95 \%$ methanol over $10 \mathrm{~s}$, hold at $95 \%$ methanol for 3 min, then immediately revert to starting conditions and hold for $3 \mathrm{~min}$. Compound $1(1 \mathrm{mg})$ eluted at $13.3 \mathrm{~min}$ to yield 6hydroxy-1,2 benzoxazol-3(2H)-one. 


\section{3,6-dihydroxy-1,2-benzisoxazole (1)}

The synthesis shown in Supplementary Fig. 1 is based on one previously published [61]. Hydroxylamine hydrochloride $(2.50 \mathrm{~g}, 36.0 \mathrm{mmol})$ was added to a stirring solution of $\mathrm{KOH}(2.69 \mathrm{~g}, 47.9 \mathrm{mmol})$ in water $(30 \mathrm{ml})$ at room temperature. Methyl 2,4-dihydroxy-benzoate (1.01 g, $6.0 \mathrm{mmol})$ dissolved in 1,4 dioxane $(5 \mathrm{ml})$ was added dropwise to the hydroxylamine solution and stirred for $24 \mathrm{~h}$ at room temperature. After removing the 1,4 dioxane in vacuo, the $\mathrm{pH}$ was adjusted to 1 using $3 \mathrm{~N} \mathrm{HCl}$ and extracted with ethyl acetate $(3 \times 50 \mathrm{ml})$. The organic layers were combined and dried over $\mathrm{Na}_{2} \mathrm{SO}_{4}$, filtered and concentrated in vacuo. The resultant residue was purified by silica gel flash chromatography using a gradient of $20-100 \%$ ethyl acetate/hexanes to afford 2,4-dihydroxybenzohydroxamic acid $(765.7 \mathrm{mg}, 76 \%$ yield).

2,4-dihydroxy-benzohydroxamic acid $\quad(161.4 \mathrm{mg}$, $0.96 \mathrm{mmol}$ ) was dissolved in anhydrous tetrahydrofuran $(10 \mathrm{ml})$ under $\mathrm{N}_{2}$. Dry 1,1'-carbonyldiimidazole (CDI) (489.2 $\mathrm{mg}, 3.0 \mathrm{mmol}$ ) was added to the mixture and heated at reflux conditions for $30 \mathrm{~min}$. $\mathrm{Et}_{3} \mathrm{~N}(210 \mu \mathrm{l}, 1.5 \mathrm{mmol})$ was then added, and the reaction proceeded at reflux for $18 \mathrm{~h}$. The reaction mixture was then left to cool to room temperature, and THF was removed in vacuo. The resulting residue was reconstituted in $1 \mathrm{~N} \mathrm{HCl}$ and extracted with EtOAc $(3 \times 50 \mathrm{ml})$. The combined organic layers were dried over $\mathrm{Na}_{2} \mathrm{SO}_{4}$, filtered and concentrated. The resulting residue was purified by silica gel flash chromatography using a gradient of $30-50 \%$ ethyl acetate with $0.1 \%$ formic acid (FA)/hexanes to afford 3,6-dihydroxy-1,2-benzisoxazole (107.7 mg, 74\% yield, overall yield 56\%). Prior to bioassay, this product was further purified by HPLC with an isocratic method of $20 \% \mathrm{MeOH}$ in $\mathrm{H}_{2} \mathrm{O}$ with $0.1 \%$ FA to afford $4.8 \mathrm{mg}$ of $\mathbf{1}$ (white powder). ${ }^{1} \mathrm{H} \mathrm{NMR}\left(500 \mathrm{MHz}, \mathrm{CD}_{3} \mathrm{OD}\right) \delta$ $6.70(1 \mathrm{H}, \mathrm{d}, J=1.9), 6.78(1 \mathrm{H}, \mathrm{dd}, J=1.9,8.7), 7.50(1 \mathrm{H}$, d, $J=8.6) .{ }^{13} \mathrm{C}$ NMR $\left(125 \mathrm{MHz}, \mathrm{CD}_{3} \mathrm{OD}\right) \delta 96.2,108.2$, $114.5,123.6,163.2,166.9$, 167.6. HRMS calcd for $\mathrm{C}_{7} \mathrm{H}_{4} \mathrm{NO}_{3}: 150.0191$. Found $[\mathrm{M}-\mathrm{H}] 150.0206$.

\section{3-hydroxy-1,2-benzisoxazole (2)}

Compound 2 was prepared from commercially available methyl salicylate (methyl 2-hydroxy-benzoate) according to the scheme used to prepare 1. 2 was further purified by semi-preparative HPLC using an isocratic method of $50 \%$ $\mathrm{MeOH}$ in $\mathrm{H}_{2} \mathrm{O}$ with $0.1 \%$ FA to afford $6.0 \mathrm{mg}$ of 2 (white powder). ${ }^{1} \mathrm{H}$ NMR $\left(500 \mathrm{MHz},\left(\mathrm{CD}_{3}\right)_{2} \mathrm{SO}\right) \delta 7.31(1 \mathrm{H}, \mathrm{t}, J=$ $7.0), 7.54(1 \mathrm{H}, \mathrm{d}, J=8.2), 7.59(1 \mathrm{H}, \mathrm{t}, J=7.9), 7.75$ $(1 \mathrm{H}, \mathrm{d}, J=8.0), 12.45(1 \mathrm{H}$, br s$) .{ }^{13} \mathrm{C}$ NMR $(125 \mathrm{MHz}$, $\left.\left(\mathrm{CD}_{3}\right)_{2} \mathrm{SO}\right) \quad \delta \quad 110.0, \quad 114.6, \quad 121.4, \quad 122.9, \quad 130.5$, 163.0, 165.4. HRMS calcd for $\mathrm{C}_{7} \mathrm{H}_{4} \mathrm{NO}_{2}$ : 134.0242. Found $[\mathrm{M}-\mathrm{H}] 134.0251$. 3-hydroxy-6-fluoro-1,2-benzisoxazole (3)

Compound 3 was prepared from commercially available methyl 2-hydroxy-4-fluoro-benzoate according to the scheme used to prepare 1. 3 was further purified by semipreparative HPLC using the method: 12 min gradient from $55-100 \% \mathrm{MeOH}$ in $\mathrm{H}_{2} \mathrm{O}$ with $0.1 \%$ FA to yield $9.1 \mathrm{mg}$ (white powder). ${ }^{1} \mathrm{H}$ NMR $\left(500 \mathrm{MHz}, \mathrm{CD}_{3} \mathrm{OD}\right) \delta 7.09(1 \mathrm{H}$, td, $J=1.5,9.1), 7.22(1 \mathrm{H}, \mathrm{dd}, J=1.5,9.1), 7.70(1 \mathrm{H}, \mathrm{dd}$, $J=5.2,8.9) .{ }^{13} \mathrm{C}$ NMR $\left(125 \mathrm{MHz}, \mathrm{CD}_{3} \mathrm{OD}\right) \delta 98.3(\mathrm{~d}, J=$ 27.7), $112.8,113.0(\mathrm{~d}, J=25.7), 123.9(\mathrm{~d}, J=11.5), 165.7$ (d, $J=14.4), 166.3$ (d, $J=249.0$ ), 167.0. HRMS calcd for $\mathrm{C}_{7} \mathrm{H}_{3} \mathrm{FNO}_{2}$ : 152.0148. Found [M $\left.-\mathrm{H}\right]:$ : 152.0271 .

\section{3-hydroxy-6-(benzyloxy)-1,2-benzisoxazole (4)}

Commercially available methyl 2,4-dihydroxy-benzoate $(1.0 \mathrm{~g}, 5.92 \mathrm{mmol})$ was dissolved in $10 \mathrm{ml}$ acetone and treated with $\mathrm{K}_{2} \mathrm{CO}_{3}(1.0 \mathrm{~g}, 7.24 \mathrm{mmol})$ and $\mathrm{NaI}(0.25 \mathrm{~g}$, $1.67 \mathrm{mmol}$ ). The resulting suspension was stirred for $10 \mathrm{~min}$ under $\mathrm{N}_{2}$ and then benzyl bromide $(700 \mu \mathrm{l}, 5.85 \mathrm{mmol})$ was added dropwise. The reaction mixture was stirred for $24 \mathrm{~h}$ at ambient temperature. After removing the acetone in vacuo, the resulting residue was resuspended in $\mathrm{H}_{2} \mathrm{O}$ and extracted with EtOAc $(4 \times 40 \mathrm{ml})$. The organic layers were combined, dried over $\mathrm{Na}_{2} \mathrm{SO}_{4}$, and concentrated in vacuo. This residue $(1.8 \mathrm{~g})$ was purified by silica gel flash chromatography using a gradient of $10-100 \%$ EtOAc in hexanes to afford methyl 2-hydroxy-4-benzyloxy-benzoate $(687.4 \mathrm{mg}, 45.5 \%$ yield) as white crystals. Methyl 2-hydroxy-4-benzyloxybenzoate was then subject to the synthetic steps used to obtain $\mathbf{1}$, which afforded $\mathbf{4}$, which was further purified by semi-preparative HPLC using an isocratic method of 50\% $\mathrm{MeOH}$ in $\mathrm{H}_{2} \mathrm{O}$ with $0.1 \%$ FA. This obtained $11.0 \mathrm{mg}$ (white powder). ${ }^{1} \mathrm{H}$ NMR $\left(500 \mathrm{MHz},\left(\mathrm{CD}_{3}\right)_{2} \mathrm{SO}\right) \delta 5.17(2 \mathrm{H}, \mathrm{s})$, $6.94(1 \mathrm{H}, \mathrm{d}, J=8.7), 7.15(1 \mathrm{H}, \mathrm{s}), 7.34(1 \mathrm{H}, \mathrm{t}, J=7.4)$, $7.40(2 \mathrm{H}, \mathrm{t}, J=7.4), 7.46(2 \mathrm{H}, \mathrm{d}, J=7.4), 7.60(1 \mathrm{H}, \mathrm{d}, J=$ 8.6). ${ }^{13} \mathrm{C}$ NMR $\left(125 \mathrm{MHz},\left(\mathrm{CD}_{3}\right)_{2} \mathrm{SO}\right) \delta 69.9,94.5,108.3$, 113.4, 122.0, 127.9 (2 C), 128.1, 128.5 (2 C), 136.5, 161.1, 164.9, 165.7. HRMS calcd for $\mathrm{C}_{14} \mathrm{H}_{10} \mathrm{NO}_{3}$ : 240.0661 . Found $[\mathrm{M}-\mathrm{H}] 240.0564$.

\section{3-hydroxy-6-amino-1,2-benzisoxazole (5)}

Compound $\mathbf{5}$ was prepared from commercially available methyl 2-hydroxy-4-amino-benzoate according to the scheme used to prepare $\mathbf{1}$ with minor modification. The ring-closing step was accomplished with $\mathrm{CDI} / \mathrm{MeCN}$ instead of $\mathrm{CDI} / \mathrm{THF} / \mathrm{Et}_{3} \mathrm{~N}$ as used for 1 . The resulting mixture was purified using semi-preparative HPLC with a gradient method from $10-55 \% \mathrm{MeOH}$ in $\mathrm{H}_{2} \mathrm{O}$ with $0.1 \%$ FA. This afforded $1.1 \mathrm{mg}$ of $\mathbf{5}$ (white powder). ${ }^{1} \mathrm{H}$ NMR $\left(500 \mathrm{MHz},\left(\mathrm{CD}_{3}\right)_{2} \mathrm{SO}\right) \delta 5.80(2 \mathrm{H}, \mathrm{s}), 6.39(1 \mathrm{H}, \mathrm{d}, J=1.4)$, 
6.50, (1H, dd, $J=1.5,8.4), 7.30(1 \mathrm{H}, \mathrm{d}, J=8.4) .{ }^{13} \mathrm{C}$ NMR $\left(125 \mathrm{MHz},\left(\mathrm{CD}_{3}\right)_{2} \mathrm{SO}\right) \delta 91.2,103.6,111.8,121.8,152.3$, 165.6, 165.9. HRMS calcd for $\mathrm{C}_{7} \mathrm{H}_{5} \mathrm{~N}_{2} \mathrm{O}_{2}: 149.0351$. Found $[\mathrm{M}-\mathrm{H}] 149.0323$.

\section{3-hydroxy-6-methoxy-1,2-benzisoxazole (6)}

Compound 6 was purchased commercially (SigmaAldrich).

\section{3,4,6-trihydroxy-1,2-benzisoxazole (7)}

Compound 7 was prepared from commercially available methyl 2,4,6-trihydroxy-benzoate according to the scheme used to prepare 1 . 7 was further purified from a mixture by semi-preparative HPLC with a gradient method of $25-100 \% \mathrm{MeOH}$ in $\mathrm{H}_{2} \mathrm{O}$ with $0.1 \%$ FA. This afforded $6.8 \mathrm{mg}$ of 7 (white powder). ${ }^{1} \mathrm{H}$ NMR $\left(500 \mathrm{MHz},\left(\mathrm{CD}_{3}\right)\right.$ $\left.{ }_{2} \mathrm{SO}\right) \delta 6.08(1 \mathrm{H}, \mathrm{d}, J=1.3), 6.14(1 \mathrm{H}, \mathrm{d}, J=1.3), 10.22$ (3H, br s). ${ }^{13} \mathrm{C}$ NMR $\left(125 \mathrm{MHz},\left(\mathrm{CD}_{3}\right)_{2} \mathrm{SO}\right) \delta 86.8,96.6$, 97.3, 153.8, 161.9, 165.2, 166.6. HRMS calcd for $\mathrm{C}_{7} \mathrm{H}_{4} \mathrm{NO}_{4}$ : 166.0140. Found [M - H] 166.0151.

\section{N-methyl-6-hydroxy-1,2-benzisoxazol-3(2H)-one (8) and $\mathrm{N}$-methyl-6-methoxy-1,2-benzisoxazol-3(2H)- one (9)}

Compounds 8 and 9 were prepared from 1 in a one-step synthesis and purification. 1 (46 $\mathrm{mg}, 0.33 \mathrm{mmol})$ was dissolved in dry $\mathrm{MeCN}(4 \mathrm{ml})$, and $\mathrm{K}_{2} \mathrm{CO}_{3}(91 \mathrm{mg}, 0.66 \mathrm{mmol})$ was suspended in the solution. Suspension was stirred rapidly under $\mathrm{N}_{2}$, and excess $\mathrm{CH}_{3} \mathrm{I}(260 \mu \mathrm{l}, 4.2 \mathrm{mmol})$ was added dropwise and stirred for $18 \mathrm{~h}$ at room temperature. The reaction was quenched by removing $\mathrm{MeCN}$ in vacuo, and the resulting residue was dissolved in $1 \mathrm{~N} \mathrm{HCl}$. This solution was extracted with EtOAc $(3 \times 30 \mathrm{ml})$, and the combined organic layers were dried over $\mathrm{Na}_{2} \mathrm{SO}_{4}$, filtered and concentrated in vacuo. The resulting products were purified by semi-preparative HPLC using a 12 min gradient from $35-80 \% \mathrm{MeOH}$ in $\mathrm{H}_{2} \mathrm{O}$ with $0.1 \%$ FA to afford 8 (12.1 mg, white powder) and $9(8.5 \mathrm{mg}$, white powder).

Compound $8{ }^{1} \mathrm{H}$ NMR $\left(500 \mathrm{MHz},\left(\mathrm{CD}_{3}\right)_{2} \mathrm{SO}\right) \delta 3.46$ $(3 \mathrm{H}, \mathrm{s}), 6.68(1 \mathrm{H}, \mathrm{d}, J=1.6), 6.57(1 \mathrm{H}, \mathrm{dd}, J=1.6,8.4)$, $7.55(1 \mathrm{H}, \mathrm{d}, J=8.4), 10.73(1 \mathrm{H}, \mathrm{br} \mathrm{s}) .{ }^{13} \mathrm{C}$ NMR $(125 \mathrm{MHz}$, $\left.\left(\mathrm{CD}_{3}\right)_{2} \mathrm{SO}\right) \delta 33.3,95.3,106.8,113.4,124.7,162.3,163.4$, 164.2. HRMS calcd for $\mathrm{C}_{8} \mathrm{H}_{8} \mathrm{NO}_{3}: 166.0504$. Found $[\mathrm{M}+$ H]: 166.0216.

Compound $9{ }^{1} \mathrm{H}$ NMR $\left(500 \mathrm{MHz},\left(\mathrm{CD}_{3}\right)_{2} \mathrm{SO}\right) \delta 3.51$ $(3 \mathrm{H}, \mathrm{s}), 3.86(3 \mathrm{H}, \mathrm{s}), 6.89(1 \mathrm{H}, \mathrm{dd}, J=2.1,8.6), 7.05(1 \mathrm{H}$, $\mathrm{d}, J=2.0), 7.64(1 \mathrm{H}, \mathrm{d}, J=8.6) .{ }^{13} \mathrm{C}$ NMR $(125 \mathrm{MHz}$, $\left.\left(\mathrm{CD}_{3}\right)_{2} \mathrm{SO}\right) \delta 33.2,56.1,93.8,108.1,113.2,124.5,162.2$, 163.7, 164.5. HRMS calcd for $\mathrm{C}_{9} \mathrm{H}_{10} \mathrm{NO}_{3}: 180.0661$. Found $[\mathrm{M}+\mathrm{H}]: 180.0341$. 6-hydroxy-2-benzoxazolinone (10)

Compound 10 was purchased commercially (abcr $\mathrm{GmbH}$ ).

\section{Biological assays}

Media Luria Bertani (LB) medium and cation-adjusted Mueller-Hinton broth (MHB) were purchased from SigmaAldrich. DM01 medium contains $25 \mathrm{mM}$ potassium phosphate, pH $6\left(3.3 \mathrm{mM} \mathrm{K} \mathrm{KPO}_{4}, 21.7 \mathrm{mM} \mathrm{KH}_{2} \mathrm{PO}_{4}\right), 1 \mathrm{mM}$ $\mathrm{MgSO}_{4}, 100 \mu \mathrm{M} \mathrm{CaCl}_{2}, 75 \mathrm{mM}$ sodium pyruvate, $1 \mathrm{gl}^{-1}$ $\mathrm{NH}_{4} \mathrm{Cl}$.

Bacterial susceptibility determinations Antimicrobial activity of marine bacterial extracts, semi-purified fractions, and pure compounds were evaluated by whole-cell assays with $E$. coli isolates expressing one of three archetype RND efflux pumps (AcrAB-TolC, MexAB-OprM, and MexXYOprM) that are known to contribute to antibiotic resistance in Enterobacteriaceae and $P$. aeruginosa clinical isolates. Each of the three E. coli isolates overexpressing RND efflux pumps were used in 96-well plate-based assays to determine bacterial susceptibility of B158 crude extract or fraction tested in duplicate at $1 \mathrm{mg} \mathrm{ml}^{-1}$ and determined by rapid $p$-iodonitrotetrazolium chloride colorimetric assay as described in [34]. Minimum inhibitory concentrations (MICs; defined as the lowest concentration that results in no visible growth) of the synthetically derived compound $\mathbf{1}$ was determined using a 2-fold standard microdilution method in Muller-Hinton broth (MHB) in microtiter plates as defined by the National Committee for Clinical Laboratory Standards [62] for each of the three RND overexpressing E. coli strains.

Pathogen strains and MIC testing A. baumannii UNT1901, A. baumannii UNT197-1, and E. coli UNT156-1 are clinical isolates kindly provided by Dr. William Weiss at North Texas University. P. aeruginosa strain UCBPP-PA14 was kindly provided by Dr. Frederick M. Ausubel. E. coli EC2 ((Migula) Castellani and Chalmers; ATCC $25922^{\mathrm{TM}}$, $K$. pneumoniae ATCC $43816^{\mathrm{TM}}$ and ATCC BAA-2146 ${ }^{\mathrm{TM}}$, and A. baumannii ATCC $19606^{\mathrm{TM}}$ were obtained from the American Type Culture Collection. A. baumannii L1051 was obtained from a patient skin sample at Rhode Island Hospital (Providence, RI) and is maintained in the LaPlante laboratory. A. baumannii, Isolate 9, NR-13382 and A. baumannii, Strain Naval-81, NR-17786 are human blood isolates obtained from the Biodefense and Emerging Infection Research Resources Repository (BEI) Resources, NIAID, NIH. L1051 and NR-13382 are considered multidrug resistant organisms (MDROs; non-susceptibility to one agent in three antimicrobial classes), according to previously described definitions $[37,38]$. Minimum inhibitory 
concentrations (MICs) were determined in duplicate by broth microdilution in accordance with Clinical Laboratory Standards Institute (CLSI) standards [63, 64].

Phenotype microarray experiments Five different types of 96-well phenotype microarray plates (PM1-PM5) were purchased from Biolog, Inc. A $2 \mathrm{ml}$ overnight bacteria culture (A. baumannii strain UNT197 or $P$. aeruginosa UCBPP14) in LB was grown in a shaking incubator at $37^{\circ} \mathrm{C}$. The culture was pelleted by centrifugation, washed, and resuspended in the same volume of phosphate-buffered saline (PBS). DM01 medium with or without $2 \mu \mathrm{g} \mathrm{ml}^{-1}$ of $\mathbf{1}$ were each inoculated with $1 / 1000$ volume of the resuspended culture. $100 \mu \mathrm{l}$ of the inoculated medium was added to each well of the phenotype microarray plates. Plates were covered and incubated at $37^{\circ} \mathrm{C}$. Plates were transferred to a Spectramax i3x plate reader (Molecular Devices) for measurement of $\mathrm{OD}_{600}$ after 17 and $23 \mathrm{~h}$.

\section{Targeted antibiotic antagonism experiments $(P$. aeruginosa} UCBPP14) A $2 \mathrm{ml}$ overnight culture of $P$. aeruginosa UCBPP14 in LB was grown in a shaking incubator at $37^{\circ} \mathrm{C}$. The culture was pelleted by centrifugation, washed, and resuspended in the same volume of phosphate-buffered saline (PBS). DM01 medium was supplemented with 2-fold serial dilutions of 4-hydroxybenzoic acid or 4hydroxybenzaldehyde (Sigma-Aldrich) both with and without $16 \mu \mathrm{g} \mathrm{ml}^{-1}$ of $\mathbf{1}$, then inoculated with $1 / 1000$ volume of the bacteria suspension. The cultures were incubated at $37^{\circ} \mathrm{C}$, and $\mathrm{OD}_{600}$ was measured at $17 \mathrm{~h}$.

Sequence alignment and homology modeling of CPL The amino acid sequence of $A$. baumannii chorismate pyruvatelyase (CPL) (GenBank: SST05187.1) was aligned with template E. coli $\mathrm{CPL}$ (PDB 1FW9), which has $85 \%$ query cover, E-value of $3 \times 10^{-41}$, and $50.35 \%$ identity. Sequence alignment was done using MUSCLE [65]. The solvent-free A. baumannii homology model was created by Modeller 9.24 and refined using Modeller 9.2's salign_2d [66, 67]. Homology model with GA341 value of 1.00 and the most negative DOPE was selected [66]. The homology model was quality assessed with PROCHECK [68]. To evaluate structural similarity in root-mean-square-deviation (RMSD), the homology model was superimposed onto the template structure using UCSF Chimera's MatchMaker [69]. To create solvated A. baumannii model, water molecules of the E. coli CPL template were combined with the superimposed $A$. baumannii model using UCSF Chimera $[69,70]$.

Molecular docking of compound 1 Compound 1 and 4-HB were docked in A. baumannii (GenBank: SST05187.1) homology model using UCSF Chimera and AutoDock Vina
[70, 71]. A grid box was used to define the search parameter [70, 71]. Ten protein-ligand models were produced and ranked by docking scores. The most negatively scored protein-ligand model containing $>3 \mathrm{H}$-bonds was selected. H-bonds and Van der Waals interactions were predicted using FindHBond and Find Clashes/Contacts function of UCSF Chimera [70, 72].

Acknowledgements RD was financially supported by the Omar Magnate Foundation Fellowship. Research reported in this publication was made possible by the use of equipment and services available through the RI-INBRE Centralized Research Core Facility, which is supported by the Institutional Development Award (IDeA) Network for Biomedical Research Excellence from the National Institute of General Medical Sciences of the National Institutes of Health under grant number P20GM103430. Additional financial support to KEW and TJM was provided by the National Institute of Allergy and Infectious Disease of the National Institutes of Health under grant number 1R21AI119311-01. Acinetobacter baumannii, Isolate 9, NR13382 and Acinetobacter baumannii, Strain Naval-81, NR-17786 were obtained through BEI Resources, NIAID, NIH.

\section{Compliance with ethical standards}

Conflict of interest The authors declare that they have no conflict of interest.

Publisher's note Springer Nature remains neutral with regard to jurisdictional claims in published maps and institutional affiliations.

Open Access This article is licensed under a Creative Commons Attribution 4.0 International License, which permits use, sharing, adaptation, distribution and reproduction in any medium or format, as long as you give appropriate credit to the original author(s) and the source, provide a link to the Creative Commons license, and indicate if changes were made. The images or other third party material in this article are included in the article's Creative Commons license, unless indicated otherwise in a credit line to the material. If material is not included in the article's Creative Commons license and your intended use is not permitted by statutory regulation or exceeds the permitted use, you will need to obtain permission directly from the copyright holder. To view a copy of this license, visit http://creativecommons. org/licenses/by/4.0/.

\section{References}

1. Qureshi ZA, Hittle LE, O'Hara JA, Rivera JI, Syed A, Shields RK, et al. Colistin-resistant acinetobacter baumannii: beyond carbapenem resistance. Clin Infect Dis. 2015;60:1295-303.

2. Dijkshoorn L, Nemec A, Seifert H. An increasing threat in hospitals: multidrug-resistant Acinetobacter baumannii. Nat Rev Microbiol. 2007;5:939-51.

3. Centers for Disease Control and Prevention. Antibiotic resistance threats in the United States, 2019. 2020. https://www.cdc.gov/ drugresistance/biggest-threats.html.

4. Munoz-Price LS, Fajardo-Aquino Y, Arheart KL, Cleary T, DePascale D, Pizano L, et al. Aerosolization of Acinetobacter baumannii in a Trauma ICU*. Crit Care Med. 2013;41:1915-8.

5. Gao J, Zhao X, Bao Y, Ma R, Zhou Y, Li X, et al. Antibiotic resistance and OXA-type carbapenemases-encoding genes in airborne Acinetobacter baumannii isolated from burn wards. Burns. 2014;40:295-9. 
6. Yakupogullari Y, Otlu B, Ersoy Y, Kuzucu C, Bayindir Y, Kayabas U, et al. Is airborne transmission of Acinetobacter baumannii possible: a prospective molecular epidemiologic study in a tertiary care hospital. Am J Infect Control. 2016;44:1595-9.

7. Boucher HW, Talbot GH, Bradley JS, Edwards JE, Gilbert D, Rice LB, et al. Bad bugs, no drugs: no ESKAPE! an update from the Infectious Diseases Society of America. Clin Infect Dis. 2009;48:1-12.

8. O'Shea MK. Acinetobacter in modern warfare. Int J Antimicrob Agents. 2012;39:363-75.

9. Goic-Barisic I, Seruga Music M, Kovacic A, Tonkic M, Hrenovic J. Pan drug-resistant environmental isolate of Acinetobacter baumannii from Croatia. Micro Drug Resist. 2016;23:494-6.

10. Leite GC, Oliveira MS, Perdigão-Neto LV, Rocha CKD, Guimarães T, Rizek C, et al. Antimicrobial combinations against panresistant Acinetobacter baumannii isolates with different resistance mechanisms. PLoS One. 2016;11:e0151270.

11. Rhomberg PR, Jones RN. Summary trends for the meropenem yearly susceptibility test information collection program: a 10year experience in the United States (1999-2008). Diagn Microbiol Infect Dis. 2009;65:414-26.

12. Maragakis LL, Perl TM. Antimicrobial resistance: acinetobacter baumannii: epidemiology, antimicrobial resistance, and treatment options. Clin Infect Dis. 2008;46:1254-63.

13. Boucher HelenW, Talbot GeorgeH, Benjamin DanielK Jr, Bradley John, Guidos RobertJ, Jones RonaldN, Murray BarbaraE, Bonomo DG RobertA. 10 x '20 Progress-development of new drugs active against gram-negative bacilli: an update from the Infectious Diseases Society of America. Clin Infect Dis. 2013;56:1685-94.

14. Deng BL, Cullen MD, Zhou Z, Hartman TL, Buckheit RW, Pannecouque C, et al. Synthesis and anti-HIV activity of new alkenyldiarylmethane (ADAM) non-nucleoside reverse transcriptase inhibitors (NNRTIs) incorporating benzoxazolone and benzisoxazole rings. Bioorg Med Chem. 2006;14:2366-74.

15. Chaker A, Najahi E, Chatriant O, Valentin A, Téné N, Treilhou $\mathrm{M}$, et al. New 3-substituted-2,1-benzisoxazoles: synthesis and antimicrobial activities. Arab J Chem. 2017;10:S2464-70.

16. Sivala MR, Chintha V, Potla KM, Chinnam S, Chamarthi NR. In silico docking studies and synthesis of new phosphoramidate derivatives of 6-fluoro-3-(piperidin-4-yl)benzo[d]isoxazole as potential antimicrobial agents. J Recept Signal Transduct. 2020; 40:1-7.

17. Shivaprasad CM, Jagadish S, Swaroop TR, Mohan CD, Roopashree R, Kumar KSS, et al. Synthesis of new benzisoxazole derivatives and their antimicrobial, antioxidant and antiinflammatory activities. Eur J Chem. 2014;5:91-5.

18. Wierenga W, Evans BR, Zurenko GE. Benzisoxazolones: antimicrobial and antileukemic activity. J Med Chem. 1984;27: $1212-5$.

19. Hrib NJ, Jurcak JG, Burgher KL, Conway PG, Hartman HB, Kerman LL, et al. Benzisoxazole- and benzisothiazole-3carboxamides as potential atypical antipsychotic agents. J Med Chem. 1994;37:2308-14.

20. Davis L, Effland RC, Klein JT, Dunn RW, Geyer HM, Petko WW. 3-substituted-1,2-benzisoxazoles: novel antipsychotic agents. Drug Des Disco. 1992;8:225-40.

21. Saunders JC, Williamson WRN. Potential antiinflammatory compounds. 2. Acidic antiinflammatory 1,2-benzisoxazoles. J Med Chem. 1979;22:1554-8.

22. Khedekar P, Bahekar R, Chopadec R, Umathec S, Rao A, Bhusaria $\mathrm{K}$. Synthesis and anti-inflammatory activity of alkyl/arylidene-2aminobenzothiazoles and 1-benzothiazol-2-yl-3-chloro-4-substituted-azetidin-2-ones. Arzneimittelforschung 2011;53:640-7.

23. Anand M, Selvaraj V, Alagar M. Synthesis, characterization and evaluation of antioxidant and anticancer activities of novel benzisoxazole-substituted-allyl derivatives. Korean J Chem Eng. 2014;31:659-63.

24. Chandra S, Mahadimane V. Effect of novel benzisoxazole derivatives against ehrlich ascites carcinoma cells in swiss albino mice: cytotoxic and haematalogical studies. Int J Pharma Sci Res. 2015;6:3606-11.

25. Jain $\mathrm{M}$, Kwon $\mathrm{CH}$. 1,2-Benzisoxazole phosphorodiamidates as novel anticancer prodrugs requiring bioreductive activation. J Med Chem. 2003;46:5428-36.

26. Ashwini N, Garg M, Mohan CD, Fuchs JE, Rangappa S, Anusha $\mathrm{S}$, et al. Synthesis of 1,2-benzisoxazole tethered 1,2,3-triazoles that exhibit anticancer activity in acute myeloid leukemia cell lines by inhibiting histone deacetylases, and inducing p21 and tubulin acetylation. Bioorg Med Chem. 2015;23:6157-65.

27. Aiello S, Wells G, Stone EL, Kadri H, Bazzi R, Bell DR, et al. Synthesis and biological properties of benzothiazole, benzoxazole, and chromen-4-one analogues of the potent antitumor agent 2(3,4-dimethoxyphenyl)-5- fluorobenzothiazole (PMX 610, NSC 721648). J Med Chem. 2008;51:5135-9.

28. Rakesh KP, Shantharam CS, Sridhara MB, Manukumar HM, Qin HL. Benzisoxazole: a privileged scaffold for medicinal chemistry. Med Chem Commun. 2017;8:2023-39.

29. Purohit SS, VeerapurV P. Benzisoxazole containing thiazolidinediones as peroxisome proliferator activated receptor- $\gamma$ agonists: design, molecular docking, synthesis \& antidiabetic studies. Sch Acad J Pharm. 2014;3:26-37.

30. Sills GJ. Zonisamide. In: Enna SJ, Bylund DB, editors. xPharm: the comprehensive pharmacology reference. University of Liverpool, Liverpool, United Kingdom: Elsevier; 2009. p. 1-6.

31. Vasquez AR, Bobo WV. Mood stabilizers: risperidone for treating bipolar disorders in adults. In: Riederer P, Laux G, Mulsant B, Le W, Nagatsu T, editors. NeuroPsychopharmacotherapy. Cham: Springer; 2020. p. 1-32.

32. Green B. Paliperidone: a clinical review. Curr Drug Ther. 2009;4: $7-11$.

33. Weiden P. Iloperidone for the treatment of schizophrenia: an updated clinical review. Clin Schizophr Relat Psychoses. 2012;6: 34-44.

34. Whalen KE, Poulson-Ellestad KL, Deering RW, Rowley DC, Mincer TJ. Enhancement of antibiotic activity against multidrugresistant bacteria by the efflux pump inhibitor 3,4-dibromopyrrole2,5-dione isolated from a pseudoalteromonas sp. J Nat Prod. 2015;78:402-12.

35. Hamada M, Kondo S, Nakamura H, Ikeda T, Ikeda D, Iinuma K, et al. A new antibiotic,3,6-dihydroxyindoxazene. J Antibiot. 1983;36:445-7.

36. Imai $\mathrm{H}$, Suzuki K, Miyazaki $\mathrm{S}$, Tanaka $\mathrm{K}$, Watanabe $\mathrm{S}$, Iwanami M. A new antibiotic Y-T0678H produced by a Chromobacterium species. J Antibiot. 1983;36:911-2.

37. Magiorakos AP, Srinivasan A, Carey RB, Carmeli Y, Falagas ME, Giske CG, et al. Multidrug-resistant, extensively drug-resistant and pandrug-resistant bacteria: an international expert proposal for interim standard definitions for acquired resistance. Clin Microbiol Infect. 2012;18:268-81.

38. Beganovic M, Luther MK, Daffinee KE, LaPlante KL. Biofilm prevention concentrations (BPC) of minocycline compared to polymyxin $\mathrm{B}$, meropenem, and amikacin against Acinetobacter baumannii. Diagnostic Microbial Infect Dis. 2019;94:223-6.

39. Uto Y. 1,2-Benzisoxazole compounds: a patent review (20092014). Expert Opin Ther Pat. 2015;25:643-62.

40. Uto Y. 1, 2-Benzisoxazole: a privileged structure with a potential for polypharmacology. Curr Pharm Des. 2016;22:3201-11.

41. Meganathan R. Ubiquinone biosynthesis in microorganisms. FEMS Microbiology Letters. 2001;203:131-9.

42. Matsushita K, Yamada M, Shinagawa E, Adachi O, Ameyama M. Function of ubiquinone in the electron transport system of 
pseudomonas aeruginosa grown aerobically1. J Biochem. 1980;88:757-64.

43. Tran UPC, Clarke CF. Endogenous synthesis of coenzyme Q in eukaryotes. Mitochondrion 2007;7:1-17.

44. Dosselaere F, Vanderleyden J. A metabolic node in action: chorismate-utilizing enzymes in microorganisms. Crit Rev Microbiol. 2001;27:75-131.

45. Herrmann KM, Weaver LM. The shikimate pathway. Annu Rev Plant Physiol Plant Mol Biol. 1999;50:473-503.

46. Siebert M, Severin K, Heide L. Formation of 4-hydroxybenzoate in Escherichia coli: characterization of the ubiC gene and its encoded enzyme chorismate pyruvate-lyase. Microbiology 1994;140:897-904.

47. Nichols BP, Green JM. Cloning and sequencing of Escherichia coli ubiC and purification of chorismate lyase. J Bacteriol. 1992;174:5309-16.

48. Siebert M, Bechthold A, Melzer M, May U, Berger U, Schröder $\mathrm{G}$, et al. Ubiquinone biosynthesis cloning of the genes coding for chorismate pyruvate-lyase and 4-hydroxybenzoate octaprenyl transferase from Escherichia coli. FEBS Lett. 1992;307:347-50.

49. Gallagher DT, Mayhew M, Holden MJ, Howard A, Kim K-J, Vilker VL. The crystal structure of chorismate lyase shows a new fold and a tightly retained product. Proteins Struct Funct Genet. 2001;44:304-11.

50. Smith N, Roitberg AE, Rivera E, Howard A, Holden MJ, Mayhew $\mathrm{M}$, et al. Structural analysis of ligand binding and catalysis in chorismate lyase. Arch Biochem Biophys. 2005;445:72-80.

51. Holden MJ, Mayhew MP, Gallagher DT, Vilker VL. Chorismate lyase: kinetics and engineering for stability. Biochim Biophys Acta Protein Struct Mol Enzymol. 2002;1594:160-7.

52. Han S-S, Kyeong H-H, Choi JM, Sohn Y-K, Lee J-H, Kim H-S. Engineering of the conformational dynamics of an enzyme for relieving the product inhibition. ACS Catal. 2016;6:8440-5.

53. Young IG, Leppik RA, Hamilton JA, Gibson F. Biochemical and genetic studies on ubiquinone biosynthesis in Escherichia coli K12:4-hydroxybenzoate octaprenyltransferase. J Bacteriol. 1972; 110:18-25.

54. Bräuer L, Brandt W, Schulze D, Zakharova S, Wessjohann L. A structural model of the membrane-bound aromatic prenyltransferase UbiA from E. coli. ChemBioChem. 2008;9:982-92.

55. Wessjohann L, Sontag B. Prenylation of benzoic acid derivatives catalyzed by a transferase fromescherichia coli overproduction: method development and substrate specificity. Angew Chem Int Ed Engl. 1996;35:1697-9.

56. Hsu AY, Poon WW, Shepherd JA, Myles DC, Clarke CF. Complementation of coq3 mutant yeast by mitochondrial targeting of the Escherichia coli UbiG polypeptide: evidence that UbiG catalyzes both $\mathrm{O}$-methylation steps in ubiquinone biosynthesis. Biochemistry. 1996;35:9797-806.
57. Marshall SA, Payne KAP, Leys D. The UbiX-UbiD system: the biosynthesis and use of prenylated flavin ( $\mathrm{prFMN})$. Arch Biochem Biophys. 2017;632:209-21.

58. Moore CM, Mills MM, Arrigo KR, Berman-Frank I, Bopp L, Boyd PW, et al. Processes and patterns of oceanic nutrient limitation. Nat Geosce. 2013;6:701-10.

59. Bax R, Green S. Antibiotics: the changing regulatory and pharmaceutical industry paradigm. J Antimicrob Chemother. 2015;70: 1281-4.

60. Maxson T, Mitchell DA. Targeted treatment for bacterial infections: prospects for pathogen-specific antibiotics coupled with rapid diagnostics. Tetrahedron. 2016;72:3609-24.

61. Fang QK, Hopkins S, Jones S. Benzo[d]isoxazol-3-ol DAAO inhibitors (U.S. Patent 20050143434A1), 2005.

62. Andrews JM. Determination of minimum inhibitory concentrations. J Antimicrob Chemother. 2001;48:5-16.

63. Patel JB, Cockerill FR, Bradford PA, Eliopoulos GM, Hindler JA, Jenkins SG, et al. M07-A10 Methods for Dilution Antimicrobial Susceptibility Tests for Bacteria That Grow Aerobically; Approved Standard-. Clinical Laboratory Standards Institute. 2015;35.

64. Clinical and Laboratory Standards Institute. M100-S25: performance standards for antimicrobial susceptibility testing; twentyfifth informational supplement. CLSI, 2015;35:1-240.

65. Edgar RC. MUSCLE: multiple sequence alignment with high accuracy and high throughput. Nucleic Acids Res. 2004;32: 1792-7.

66. Šali A, Blundell TL. Comparative protein modelling by satisfaction of spatial restraints. J Mol Biol. 1993;234:779-815.

67. Braberg H, Webb BM, Tjioe E, Pieper U, Sali A, Madhusudhan MS. SALIGN: a web server for alignment of multiple protein sequences and structures. Bioinformatics. 2012;28:2072-3.

68. Laskowski RA, MacArthur MW, Moss DS, Thornton JM. PROCHECK: a program to check the stereochemical quality of protein structures. J Appl Crystallogr. 1993;26:283-91.

69. Meng EC, Pettersen EF, Couch GS, Huang CC, Ferrin TE. Tools for integrated sequence-structure analysis with UCSF Chimera. BMC Bioinforma. 2006;7:1-10.

70. Pettersen EF, Goddard TD, Huang CC, Couch GS, Greenblatt DM, Meng EC, et al. UCSF Chimera - a visualization system for exploratory research and analysis. J Comput Chem. 2004;25: $1605-12$

71. Trott O, Olson AJ. AutoDock Vina: improving the speed and accuracy of docking with a new scoring function, efficient optimization, and multithreading. J Comput Chem. 2010;31:455-61.

72. Mills JEJ, Dean PM. Three-dimensional hydrogen-bond geometry and probability information from a crystal survey. J Comput Aided Mol Des. 1996;10:607-22. 